\title{
Erratum: "Superdiffusion of Cosmic Rays: Implications for Cosmic Ray Acceleration" (2014, ApJ, 784, 38)
}

\author{
A. Lazarian ${ }^{1}$ and Huirong Yan $^{2,3}$ (i) \\ ${ }^{1}$ Department of Astronomy, University of Wisconsin, 475 North Charter Street, Madison, WI 53706, USA \\ 2 DESY, Platanenallee 6, D-15738 Zeuthen, Germany \\ ${ }^{3}$ Institut für Physik und Astronomie, Universität Potsdam, D-14476 Potsdam-Golm, Germany \\ Received 2019 October 21; published 2019 November 12
}

An error is found in Table 1 of the published article describing the regimes of diffusion and superdiffusion. For the weak turbulence when the scales $s$ measured along the magnetic field are larger then the turbulence injection scale $L$, the separation of magnetic field lines increases in a diffusive way with the step $L M_{\mathrm{A}}^{4}$, where $M_{\mathrm{A}}$ is the Alfvén Mach number, i.e., $M_{\mathrm{A}}=V_{L} / V_{\mathrm{A}}$, where $V_{L}$ is the turbulent injection velocity and $V_{\mathrm{A}}$ is the Alfvén velocity. We provide Table 1 with the error corrected here.

The separation of magnetic field lines was studied in Lazarian \& Vishniac (1999). There it was found there that for the weak turbulence at scales $s \gg L$, where $s$ is measured along the magnetic field and $L$ is the injection scale of turbulence the separation is growing as a random walk with the step $L M_{\mathrm{A}}^{4}$, where $M_{\mathrm{A}}$ is the Alfvén Mach number. This result was used in further publications, e.g., in Lazarian (2006) to study the propagation of heat in clusters of galaxies. Unfortunately, due to an error the corresponding entry in our published article is incorrect. We provide the corrected version of the Table 1 here describing the separation of magnetic field lines for different regimes of MHD turbulence.

Table 1

Regimes of MHD Turbulence and Magnetic Field Separation

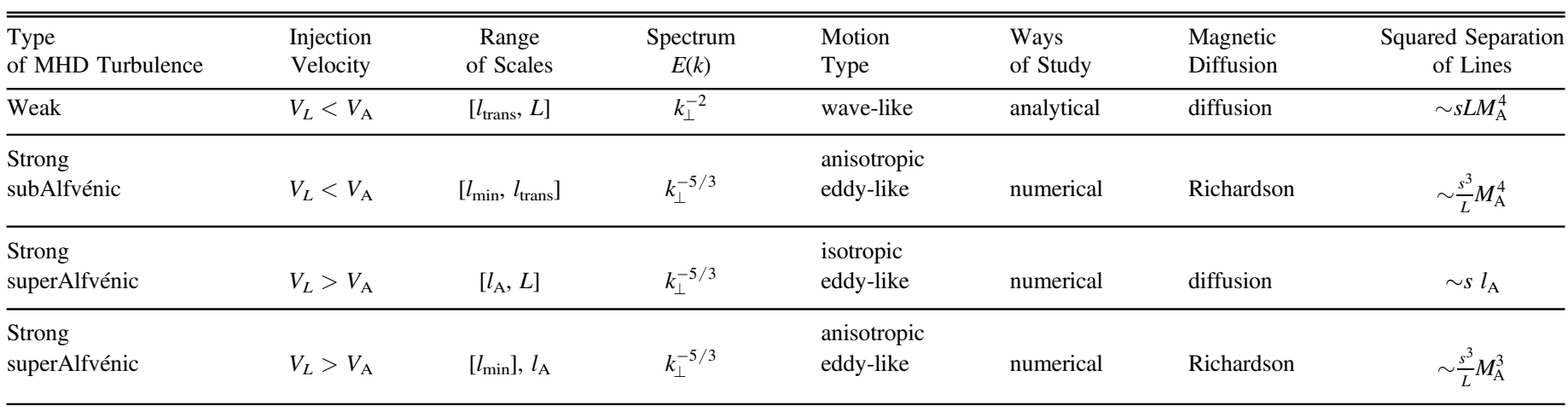

Note. $L$ and $l_{\min }$ are the injection and perpendicular dissipation scales, respectively. $M_{\mathrm{A}} \equiv \delta B / B, l_{\text {trans }}=L M_{\mathrm{A}}^{2}$ for $M_{\mathrm{A}}<1$ and $l_{A}=L M_{\mathrm{A}}^{-3}$ for $M_{\mathrm{A}}<1$. For weak Alfvénic turbulence $\ell_{\|}$does not change. $s$ is measured along magnetic field lines.

\section{ORCID iDs}

Huirong Yan ำ https://orcid.org/0000-0003-2560-8066

\section{References}

